\title{
Explorando Dados Ligados através de um Sistema de Recomendação Educacional
}

\author{
Crystiam Kelle Pereira ${ }^{1}$, Fernanda Campos ${ }^{1}$, Victor Ströele ${ }^{1}$, José Maria N. \\ David $^{1}$, Regina Braga ${ }^{1}$ \\ ${ }^{1}$ Departamento de Ciência da Computação - Universidade Federal de Juiz de Fora - \\ Juiz de Fora - MG - Brasil \\ crystiamkelle@gmail.com, fernanda.campos@ufjf.edu.br, \\ victor.stroele@ice.ufjf.br, jose.davideufjf.edu.br, \\ regina.bragadufjf.edu.br
}

\begin{abstract}
BROAD-RSI Project is a Recommender System Based on Social Interactions which wishes to accomplish educational resources recommendations consistent with the student profile and context. Both the profile and the context are automatically extracted from Collaborative Systems such as Social Networks and Virtual Learning Environments. This paper presents an extension of the RSI-BROAD project that uses Linked Data as a location source of educational resources.
\end{abstract}

Resumo. O projeto BROAD-RSI é um Sistema de Recomendação Baseado nas Interações Sociais que pretende realizar recomendações de recursos educacionais coerentes com o perfil e o contexto do aluno. Tanto o perfil, quanto o contexto são extraídos automaticamente de Sistemas Colaborativos, tais como Redes Sociais e Ambientes Virtuais de Aprendizagem. O presente trabalho apresenta uma evolução do projeto BROAD-RSI utilizando dados ligados como fonte de localização dos recursos educacionais.

\section{Introdução}

Muitos esforços recentes na área de Tecnologias na Educação têm sido feitos no sentido de contribuir com a publicação, catalogação, descoberta e recomendação de recursos educacionais. Busca-se, através de diversas propostas e técnicas diferentes [Fritzen et al. 2012], [Fernandes and Siqueira 2013][Casagrande et al. 2013][Casagrande et al. 2013] criar um ambiente educacional personalizado e mais atraente para o aluno, que desperte o seu interesse acerca de uma variedade de temas.

Dentre os muitos desafios desta área, a localização dos recursos educacionais ganha relevância se considerarmos que muitos deles estão armazenados em repositórios de objetos de aprendizagem, sendo que esses repositórios, muitas vezes, possuem acesso restrito, não estão integrados e podem adotar diferentes padrões de catalogação. Além disso, os recursos educacionais, muitas vezes, são mal descritos, com preenchimento precário dos metadados, tornando a sua localização um processo complexo e custoso para o usuário [De Santiago and Raabe 2010].

Os sistemas de recomendação (SR) têm um papel importante para auxiliar usuários que procuram algum tipo de recurso educacional a encontrarem aqueles que sejam relevantes e pertinentes ao seu perfil e ao contexto em que estão inseridos. Segundo 
[Burke 2002], um SR pode ser "qualquer sistema que produza recomendações individualizadas ou que tenha o efeito de guiar o usuário de forma personalizada para objetos do seu interesse ou que lhes sejam úteis dentre diversas opções possíveis".

As dificuldades de localização dos recursos educacionais, bem como as restrições dos repositórios de objetos de aprendizagem, têm grande impacto na eficiência dos sistemas de recomendação e de personalização de conteúdo educacional, uma vez que esses dependem da quantidade e da qualidade dos recursos educacionais disponíveis. Nesse sentido, buscamos explorar uma solução para a recomendação de recursos educacionais, visando amenizar as limitações dos repositórios de objetos de aprendizagem, através do uso de Dados Ligados como fonte de busca de recursos educacionais. O termo Dados Ligados (ou Linked Data) se refere a um conjunto de práticas para a publicação e conexão de dados estruturados na Web [Bizer et al. 2009].

O objetivo desta pesquisa é apresentar uma evolução do projeto BROAD-RSI [PEREIRA et al. 2014a], utilizando Dados Ligados como fonte complementar de conteúdo educacional. O projeto BROAD-RSI, parte do projeto BROAD [Campos et al. 2012], é um Sistema de Recomendação Baseado nas Interações Sociais que realiza recomendações de recursos educacionais coerentes com o perfil e o contexto do aluno, extraídos automaticamente de Redes Sociais.

O artigo está organizado da seguinte forma: na seção 2 é feita uma fundamentação teórica sobre Dados Ligados; na seção 3 são apresentados alguns trabalhos relacionados; na seção 4 é dada uma visão geral da arquitetura de recomendação, nomeada BROADRSI, e um detalhamento da abordagem de recomendação usando dados ligados; na seção 5 são apresentados os resultados da avaliação da proposta e, por fim, na seção 6 são feitas as considerações finais.

\section{Dados ligados}

O termo Dados Ligados pode ser sintetizado como o uso da Web para criar ligações entre os dados originários de diferentes fontes, sendo que esses dados podem estar armazenados em diversos bancos de dados, mantidos por diferentes organizações e distribuídos em diferentes localizações geográficas. Um dos principais objetivos dos Dados Ligados é estender a Web que conhecemos, para uma Web onde os dados possam estar diretamente ligados, sem necessidade da intervenção de alguma aplicação que faça essa ligação. Esta extensão da Web também é conhecida por Web de Dados [Bizer et al. 2009].

O RDF é o padrão básico da proposta de Dados Ligados, através do qual os recursos são descritos, por meio de uma ou mais assertivas sobre eles; conteúdos são publicados de forma estruturada e esses recursos podem estar interconectados através de links, diferentes dos convencionais. Esses links expressam a natureza "semântica" da ligação entre os recursos que estão sendo ligados [Bizer et al. 2009]. A SPARQL (Simple Protocol and RDF Query Language) (http://www.w3.org/TR/rdf-sparql-query/) é a linguagem de consulta para RDF e, geralmente, para os Dados Ligados. Ela é projetada para consultar as representações de dados baseados em grafos. As consultas podem ser realizadas em SPARQL Endpoints que são serviços REST que recebem as consultas e retornam seus resultados. 


\section{Trabalhos relacionados}

Estão relacionados a esta pesquisa dois grupos de trabalhos: (1) sistemas de recomendação que usam informações contextuais do usuário, extraídas de sistemas colaborativos, para recomendar conteúdo educacional; e (2) sistema de recomendação que usam Dados Ligados como fonte de busca de conteúdo educacional.

Em [Fritzen et al. 2012] é apresentada uma proposta que busca modelar o contexto a partir de mensagens de redes sociais, e usar esse contexto para melhorar a relevância na recuperação de documentos na Web, tendo como objetivo apoiar a aprendizagem colaborativa. [Fernandes and Siqueira 2013] apresentam uma abordagem para melhorar o entendimento e conhecimento dos interesses e preferências dos usuários a partir de informações disponíveis em sistemas colaborativos, mais especificamente redes sociais. A proposta é extrair automaticamente um perfil do usuário, sendo para isso consideradas as postagens dos usuários nas redes sociais. Esse trabalho não tem como objetivo principal a recomendação, no entanto menciona essa possibilidade. [Casagrande et al. 2013] apresentam uma técnica de recomendação baseada na construção implícita de perfis de usuários através da observação dos valores de metadados dos conteúdos que esses usuários acessam.

O aplicativo Talis Aspire Comunidade Edition [Heath et al. 2012] é um dos sistemas de recomendação que usam a abordagem de Dados Ligados. Esta aplicação permite que professores de universidades do Reino Unido criem e gerenciem listas de livros on-line para os cursos que lecionam. Estas listas são organizadas de tal forma que os metadados usados são padronizados e disponibilizados como Dados Ligados e os itens semelhantes são ligados uns aos outros. Feita essa organização e representação, o aplicativo consegue buscar itens semelhantes, a partir de propriedades de ligação dos dados, fornecendo a recomendação de itens semelhantes aos que estão sendo consultados. Outro exemplo de aplicativo, disponível no Facebook, que se beneficia com as conexões possíveis com Dados Ligados é "Course Profile" (http://apps.facebook.com/courseprofiles/) da Open University. Ele usa o catálogo de cursos da Open University como fonte de informações, catálogo esse que foi armazenado usando a abordagem de Dados Ligados, e permite que um usuário se conecte a outros usuários, indicando se eles já cursaram, estão cursando ou têm interesse em cursar um determinado curso. A partir da indicação de pessoas torna-se possível também sugerir outras atividades ou cursos ligados.

O modelo de recomendação abordado neste artigo busca se beneficiar de informações geradas espontaneamente pelos usuários em redes sociais para extrair características de perfil e de contexto do usuário, que são os dados de entrada do sistema de recomendação. Tais dados são relacionados às características e conexões dos recursos educacionais, representadas em um formado de dados ligados, buscando-se dessa forma selecionar recursos educacionais relevantes aos usuários, oferecendo, assim, recomendações individualizadas e personalizadas.

\section{Projeto BROAD-RSI}

O projeto BROAD-RSI engloba a definição do perfil e do contexto educacional e social dos usuários, através da aquisição semiautomática de informações disponibilizadas em redes sociais e ambientes virtuais de aprendizagem. A proposta prevê ainda a recomendação no contexto de redes sociais, levando orientações e recursos educacionais 
para os usuários fora de um ambiente acadêmico [PEREIRA et al. 2014a][PEREIRA et al. 2014b][PEREIRA et al. 2014c](Figura 1).

O diagrama de recomendação do BROAD-RSI pode ser dividido em 3 etapas principais: Extração e Representação do Perfil e do Contexto do Usuário; Aplicação Inteligente para Recomendação; e Recuperação de Recursos Educacionais.

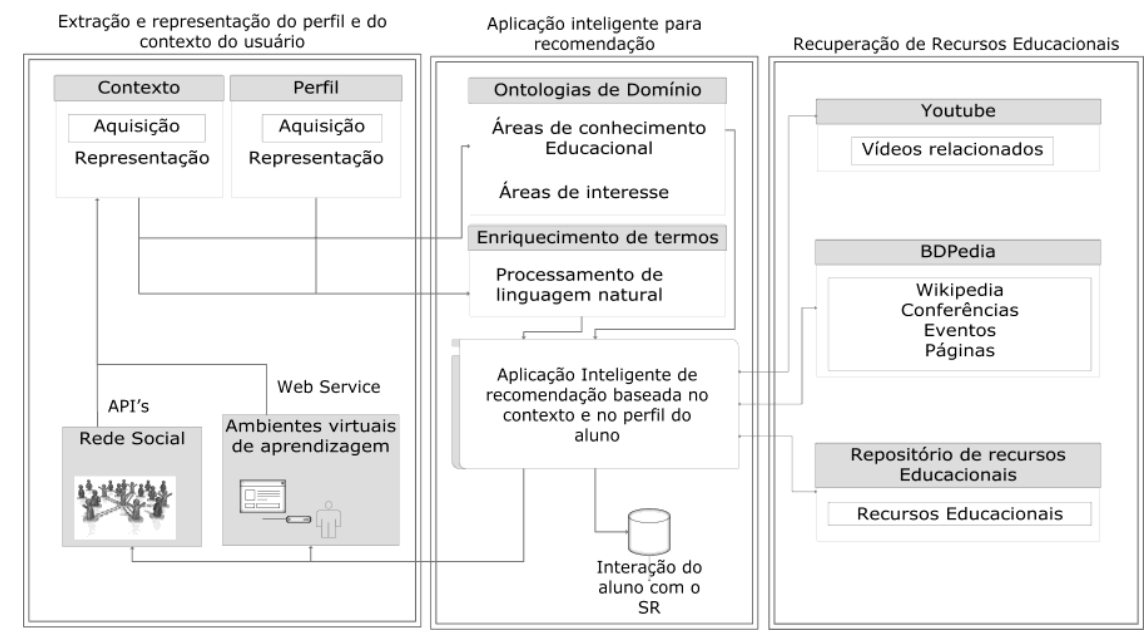

Figura 1 - Diagrama de recomendação.

A principal técnica de recomendação usada no BROAD-RSI é a técnica baseada em Conhecimento, que considera o conhecimento de como esses itens podem atender às necessidades e preferências do usuário para selecionar conteúdo que seja relevante para ele [Burke 2002]. Em um sistema de recomendação que utiliza essa técnica, a grande preocupação que se deve ter é exatamente a aquisição do conhecimento do usuário, ou seja, estabelecer estratégias para a aquisição de conhecimentos dos usuários, que são os dados de entrada usados pelo Sistema de Recomendação para selecionar e indicar itens apropriados para cada usuário.

No contexto educacional, existem muitos sistemas colaborativos que podem ser fontes importantes para a identificação de necessidades, interesses e preferências dos usuários. Dentre esses sistemas podemos destacar os Ambientes Virtuais de Aprendizagem (AVAs) e as Redes Sociais. A proposta de recomendação do BROADRSI engloba a extração de interesses educacionais tanto em AVAs, quanto em Redes Sociais. A seguir será detalhado o processo de extração para a Rede Social Facebook.

A extração do perfil e do contexto do usuário é feita através do uso da API (Application Programming Interface) disponibilizada pelo Facebook. Após a extração é feita a representação semântica do perfil e do contexto extraídos. São considerados dados de perfil: nome, email, idade, cidade de residência, idiomas de domínio, preferências por determinados tipos de mídias e escolaridade. Já o contexto engloba os cursos nos quais o usuário está matriculado, os interesses educacionais, grupos dos quais ele participa, dias e horários de uso de cada um dos sistemas.

Buscando o fortalecimento e a consistência dos interesses do usuário, que serão a informação principal do sistema de recomendação, foi necessário realizar uma etapa de enriquecimento de contexto. Ela é responsável por buscar interesses implícitos relacionados com as informações extraídas. O Enriquecimento de Contexto fez-se necessário nos tópicos de interesse, nos grupos e nos itens compartilhados, uma vez que 
eles apresentam textos em um formato livre e não detêm nenhuma estruturação estabelecida que permita a identificação dos interesses.

Para realizar o enriquecimento do contexto, foi usada a API AlchemyAPI8. Essa API baseia-se na aplicação de processamento estatístico de linguagem natural e de algoritmos de aprendizagem de máquina para a análise do conteúdo e extração de metadados semânticos, fornecendo assim, um conjunto de ferramentas para análise de conteúdo e anotações. O uso dessa API permite inferir conceitos e tópicos de interesse do usuário que não estão explícitos no perfil do usuário, nas Redes Sociais, mas que podem ser inferidas a partir da descrição de itens compartilhados.

Uma vez tendo as informações a respeito dos interesses e preferências do usuário armazenadas, o BROAD-RSI é responsável por realizar buscas em fontes diferentes, usando APIs e serviços distintos, estabelecendo a relação entre as características e preferências do usuário e os recursos educacionais armazenados nessas fontes.

Inicialmente o foco da recomendação do projeto BROAD-RSI foram os objetos de aprendizagem, armazenados em seu próprio repositório, e catalogados, através de um conjunto de metadados, conforme detalhado em [Campos et al. 2012]. No entanto, alguns problemas e limitações foram percebidos nessa abordagem, tais como a dificuldade de atualização, a especificidade dos objetos de aprendizagem, falhas na catalogação, quantidade restrita de objetos de aprendizagem, entre outras. Buscando minimizar esses problemas, a proposta de recomendação do BROAD-RSI foi expandida para incluir outras fontes de consulta de recursos educacionais, que mesmo não estando catalogados e bem caracterizados a respeito dos seus objetivos educacionais, podem contribuir com a busca por diferentes fontes de conhecimento e complementar a recomendação feita com o repositório de objetos de aprendizagem.

Com o objetivo de buscar outras fontes de recursos educacionais, além do repositório de objetos de aprendizagem, é apresentada a seguir a abordagem de recomendação utilizando duas fontes diferentes para enriquecimento das recomendações educacionais do BROAD-RSI que disponibilizam seus dados seguindo os princípios de Dados Ligados: DBPEDIA e o repositório de recursos educacionais da Open University.

\subsection{Abordagem de Recuperação de Recursos Educacionais usando Dados Ligados}

Segundo [Aquin 2012], a vantagem de depender de Dados Ligados para aplicações de recomendação e personalização é que, através do seu uso, torna-se possível obter ricas representações dos recursos e de elementos potencialmente conectados, que podem ser originários de diferentes repositórios e fontes de informação. Outra vantagem é que, abstraindo das especificidades dos sistemas e formatos em que os recursos estão armazenados, os Dados Ligados fornecem uma maneira de integrar de forma homogênea diversos recursos heterogêneos.

O uso de dados ligados na recomendação aumenta a possibilidade de encontrar conteúdos educacionais em diferentes fontes que estão sendo constantemente atualizadas, evitando o problema de escassez de novas recomendações.

Alguns repositórios estão começando a disponibilizar seus recursos educacionais no formato de Dados Ligados, ampliando as possibilidades de localização e recomendação de recursos educacionais em um domínio amplo de interesses [Dietze et 
al. 2013]. Dentre as iniciativas de disponibilização de conteúdo através de Dados Ligados, destacam-se a Open University, uma das pioneiras em disponibilizar acesso ao seu acervo através de Dados Ligados, e as Universidades Britânicas de Southampton e de Oxford.

A DBPEDIA foi escolhida por três motivos: (1) a grande quantidade de informações disponíveis; (2) a possibilidade de recuperar informação passando termos em português como parâmetros de busca; (3) a diversidade de temas para os quais são disponibilizadas informações. Já a Open University traz a vantagem de disponibilizar conteúdo com objetivo especificamente educacional, o que não acontece com a DBPEDIA.

\subsubsection{Conjunto de dados da DBPedia}

A DBPEDIA basicamente transforma qualquer página na Wikipedia em uma entidade de Dados Ligados, analisando o "infobox" na página da Wikipedia para extrair informações estruturadas sobre cada entidade e sua relação com outras entidades. Ela oferece informações gerais facilmente reutilizáveis e o poder de consulta em diferentes domínios.

O primeiro passo para a extração de recomendações educacionais através da DBPEDIA foi a busca por páginas relacionadas aos temas de interesse do usuário. Para isso foi feita uma busca usando a propriedade rdfs:label. Os rótulos de um recurso da DBPEDIA são criados a partir dos títulos das páginas da Wikipedia. Na figura 2 é apresentada uma consulta por um recurso relacionado ao interesse do aluno em Engenharia de Software.

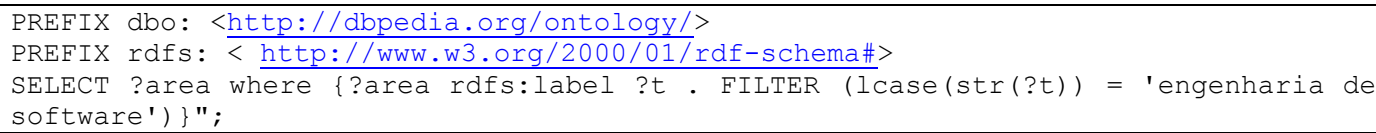

Figura 2- Sparql executada no EndPoint da DBPedia

Uma vez realizada a consulta, o serviço da DBPEDIA (http://dbpedia.org/sparql) retornará todos os recursos cujo rótulo contenha o termo indicado. Depois de recuperado o recurso, o BROAD-RSI é responsável por realizar outras consultas, por propriedades específicas que possam trazer conteúdos educacionais relevantes ao tema de interesse. Dentre as propriedades disponíveis, estão sendo utilizadas neste trabalho as seguintes: dbpedia-owl:abstract, dbpedia-owl:wikiPageExternalLink, foaf:isPrimaryTopicOf, dbpedia-owl:academicDiscipline, dbpprop:discipline of, dbpedia-owl:literaryGenre, dcterms:subject. A escolha dessas propriedades foi feita após uma pesquisa a respeito de quais propriedades retornam um conteúdo educacional útil ao usuário.

Usando essas propriedades, são recuperados e apresentados para o usuário algumas informações que podem contribuir com a sua aprendizagem, como por exemplo uma breve explicação sobre o assunto, livros, links para conferências, periódicos e outras páginas diversas que estejam associadas ao tema de interesse. Na figura 4 é mostrada uma tela do BROAD-RSI com o conteúdo educacional apresentado para o usuário, referente ao seu interesse por Engenharia de Software (esquerda).

\subsubsection{Conjunto de dados da Open University}

Um processo semelhante é utilizado para a busca de recursos educacionais do repositório da Open University. No entanto, nesse caso foi possível usar a propriedade $r d f$ s:type para agrupar os recursos apresentando-os ao usuário em grupos, tais como Livros, Podcasts, Documentos em geral, Vídeos, Cursos, etc. Na figura 3 é mostrada a SPARQL executada 
no serviço da Open University (http://data.open.ac.uk/query) para recuperar recursos do tipo Book. Na Figura 4 (direita) são mostrados os resultados da busca no repositório pelo termo "Project Management", um dos interesses do usuário.

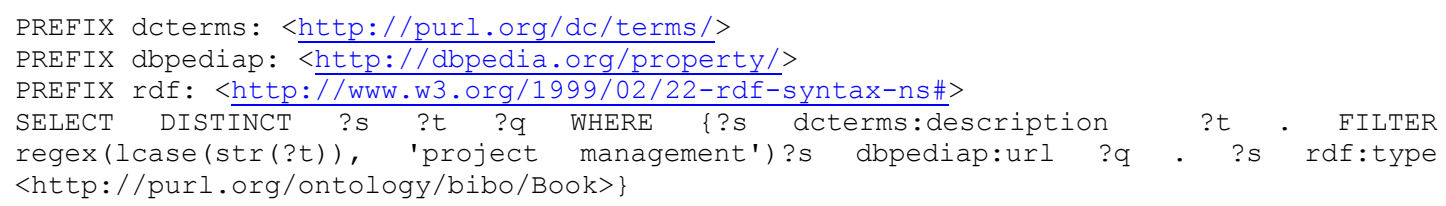

Figura 3- Sparql executada no EndPoint da DBPedia

Os recursos recuperados trazem um conjunto de outros dados ligados que podem ser úteis ao usuário. Por exemplo, um recurso do tipo Book retornado pode ser parte de um curso ou estar associado a outro recurso de outro tipo e, dessa forma, despertar o interesse em conhecer conteúdos que vão além dos recuperados pelo BROAD-RSI.

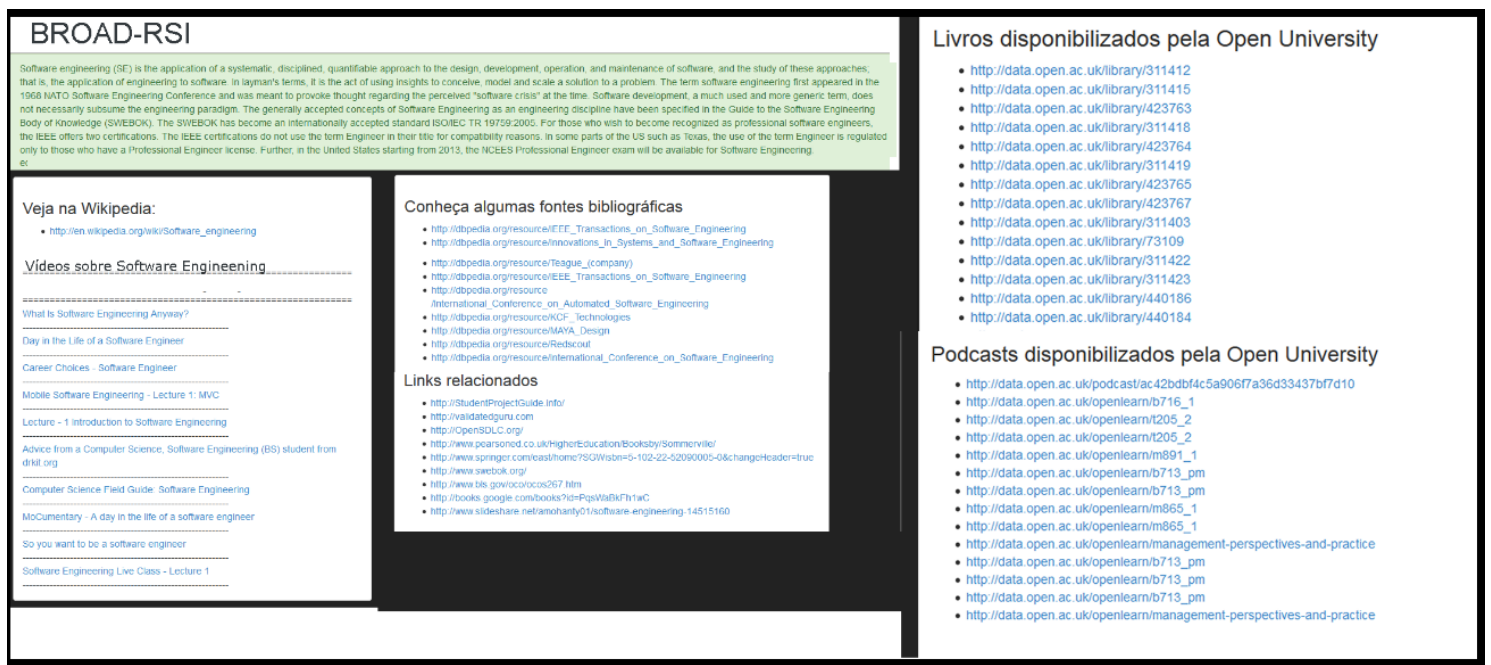

Figura 4 - Recursos recomendados a partir de conjuntos de dados ligados.

\section{Avaliação}

A avaliação da proposta apresentada foi feita através de um estudo de caso [Wohlin et al. 2012] [Yin 2001], realizado com 14 participantes voluntários, estando eles distribuídos entre estudantes de mestrado na área da Ciência da Computação, e profissionais da mesma área (analistas de sistema e professores). Um requisito para a seleção dos indivíduos foi possuir uma conta na rede social Facebook e expressar interesses através das suas interações nessa rede social. Vale ressaltar que a escolha dos indivíduos levou em consideração a área da Computação, por que o repositório de objetos de aprendizagem usado como uma das fontes para as recomendações contém catalogados recursos específicos para essa área.

O objetivo deste estudo de caso foi definido de acordo com a abordagem Goal/Question/Metric (GQM) [Basili 1992], a citar: analisar as recomendações educacionais através do BROAD-RSI para o propósito de avaliar as recomendações e comparar as duas abordagens, baseada em repositórios de objetos de aprendizagem e baseada em dados ligados, com relação à relevância das recomendações do ponto de vista do usuário no contexto de recomendações geradas a partir do BROAD-RSI para usuários da rede social Facebook. 
Para realizar a coleta dos dados os participantes do estudo de caso foram convidados a utilizar o protótipo do BROAD-RSI com o objetivo de avaliar as recomendações educacionais geradas de acordo com os interesses extraídos da rede social Facebook.

As recomendações apresentadas ao usuário estavam relacionadas a cada interesse extraído da rede social do usuário e foram divididas em dois grupos: recomendações de objetos de aprendizagem, armazenados em um repositório de objetos de aprendizagem e recomendações de recursos educacionais (links, livros, vídeos, artigos, entre outros) originadas do conjunto de dados ligados da DBPEDIA. O repositório de objetos de aprendizagem usado pelo BROAD-RSI tinha, no momento da avaliação, 60 recursos educacionais relacionados às áreas de engenharia de software, redes de computadores, computação gráfica e banco de dados. Na tabela 1 são apresentadas algumas características desses dois grupos.

Tabela 1 - Comparativo entre as duas abordagens

\begin{tabular}{|c|c|c|}
\hline & Repositório de objetos de aprendizagem & Dados Ligados \\
\hline Atualização & Atualizado com pouca frequência & $\begin{array}{l}\text { Atualizado com grande frequência } \\
\text { (depende do conjunto de dados } \\
\text { ligados) }\end{array}$ \\
\hline $\begin{array}{ll}\text { Priorização } & \text { da } \\
\text { recomendação } & \end{array}$ & $\begin{array}{l}\text { Mais precisa em virtude dos metadados } \\
\text { específicos }\end{array}$ & Pouco precisa \\
\hline $\begin{array}{l}\text { Especificidade de } \\
\text { tema }\end{array}$ & Muito específico & $\begin{array}{l}\text { Dependente do } \quad \text { conjunto } \\
\text {-DBPEDIA: pouco específica } \\
\text {-Open University: mais específica }\end{array}$ \\
\hline $\begin{array}{ll}\text { Especificidade } & \text { de } \\
\text { tipo de recurso } & \end{array}$ & Pouco específico & $\begin{array}{lcr}\text { Dependente do } & \text { conjunto } \\
\text {-DBPEDIA: pouco } & \text { específica } \\
\text {-Open University: mais específica }\end{array}$ \\
\hline Catalogação & Boa estruturação & Boa estruturação \\
\hline $\begin{array}{l}\text { Principais } \\
\text { vantagens }\end{array}$ & $\begin{array}{l}\text { Catalogação geralmente feita por } \\
\text { profissionais da educação; Recursos } \\
\text { específicos para fins educacionais; } \\
\text { Descrição detalhada através de } \\
\text { metadados; }\end{array}$ & $\begin{array}{l}\text { Diversidade de temas; Diversidade de } \\
\text { mídias; Atualização constante (em } \\
\text { alguns casos); } \quad \text { Estruturação } \\
\text { semântica; }\end{array}$ \\
\hline $\begin{array}{l}\text { Principais } \\
\text { desvantagens }\end{array}$ & $\begin{array}{l}\text { Dificuldade de atualização; } \\
\text { Repositórios específicos } \\
\text { determinadas instituições. }\end{array}$ & $\begin{array}{l}\text { Nem sempre o conteúdo tem fins } \\
\text { educacionais; } \\
\text { Falta de metadados na descrição dos } \\
\text { recursos. }\end{array}$ \\
\hline
\end{tabular}

As recomendações foram analisadas em relação à sua relevância, usando a métrica Precision [Shani and Gunawardana 2011]. Os participantes foram convidados a responder a seguinte pergunta: considerando que você está buscando recursos educacionais a respeito deste tema, esta recomendação é relevante para você?

O resultado das avaliações das recomendações, aplicando a métrica Precision, é apresentado na tabela 2, separado por cada um dos grupos de recomendação. Foram analisadas 188 recomendações, sendo 84 do Grupo 1 e 104 do Grupo 2.

Tabela 2 - Resultados da avaliação das recomendações.

\begin{tabular}{|l|l|l|}
\hline & Grupo 1 & Grupo 2 \\
\hline Concordo Plenamente & 0,39 & 0,55 \\
\hline Concordo Parcialmente & 0,19 & 0,18 \\
\hline Não concordo, nem discordo & 0,14 & 0,13 \\
\hline Discordo Parcialmente & 0,08 & 0,02 \\
\hline Discordo Plenamente & 0,19 & 0,13 \\
\hline
\end{tabular}


Através da avaliação foi possível perceber que as recomendações de recursos educacionais, cuja fonte era um conjunto de dados ligados, receberam boa aceitação dos usuários, sendo avaliada com índice um pouco superior que as recomendações do repositório de objetos de aprendizagem, mostrando-se, assim, uma possível fonte complementar de recursos educacionais a serem explorada no BROAD-RSI.

A abordagem de recomendação usando dados ligados trouxe, ainda, como vantagens a obtenção de representações ricas dos recursos e das conexões estabelecidas entre eles, além da independência em relação à localização dos repositórios. Considerando o crescimento no número de repositórios que estão disponibilizando os seus recursos educacionais usando a abordagem de dados ligados, as recomendações tendem a aumentar e serem cada vez mais diversificadas, não sofrendo os impactos da pouca atualização de um repositório de objetos de aprendizagem.

\section{Considerações Finais}

O presente trabalho apresentou uma proposta de recomendação de recursos educacionais, usando informações contextuais extraídas de redes sociais para identificar características, interesses e preferências dos alunos e usando Dados Ligados como fonte de localização dos recursos educacionais.

Apesar do foco do BROAD-RSI ser a recomendação de recursos educacionais, a utilização de dados ligados abre possibilidade de expansão do BROAD-RSI para uma abordagem de aprendizado não formal. Através das redes sociais é possível capturar interesses diversos, como, por exemplo, interesses por um determinado artista, um estilo musical, um livro, um filme, etc e, usando a abordagem tradicional do BROAD-RSI, que faz consultas, exclusivamente, em repositórios de recursos educacionais, dificilmente a recomendação conseguiria ser eficaz para interesses não educacionais. Com a evolução proposta neste trabalho, gerar recomendações para diferentes interesses seriam possível, sendo necessário para isso fazer algumas poucas modificações em relação às propriedades usadas na busca.

A pesquisa em torno do projeto BROAD-RSI está em andamento e, no âmbito do uso de Dados Ligados na recomendação, tem como passos futuros: (1) ampliar propriedades usadas para a busca de conteúdo educacional na DBPedia; (2) inclusão de outras fontes de dados ligados; (3) aprimoramento da recomendação usando avaliação de pessoas com interesses semelhantes.

\section{Agradecimentos}

Esta pesquisa é parcialmente apoiada pela UFJF, FAPEMIG, CAPES e CNPq.

\section{Referências}

Aquin, M. D. (2012). Linked Data for Open and Distance Learning. n. June.

Basili, V. R. (1992). Software modeling and measurement: the Goal/Question/Metric paradigm. Quality. http://portal.acm.org/citation.cfm?id=137076.

Bizer, C., Heath, T. and Berners-lee, T. (2009). Linked Data - The Story So Far. International Journal on Semantic Web and Information Systems (IJSWIS), v. 5, n. 3, p. $1-22$. 
Burke, R. (2002). Hybrid Recommender Systems: Survey and Experiments. User modeling and user-adapted interaction, v. 12, n. 4, p. 331-370.

Campos, F., Braga, R., Nery, T. and Santos, N. (2012). Rede de Ontologias: apoio semântico a linha de produtos de objetos de aprendizagem. Anais do Simpósio Brasileiro de Informática na Educação, v. 23, n. Sbie, p. 26-30.

Casagrande, M. F. R., Kozima, G. and Willrich, R. (22 nov 2013). Técnica de Recomendação Baseada em Metadados para Repositórios Digitais Voltados ao Ensino. XXIV Simpósio Brasileiro de Informática na Educação (SBIE 2013), v. 24, p. 677-686.

De Santiago, R. and Raabe, A. (2010). Architecture for learning objects sharing among learning institutions-LOP2P. IEEE Transactions on Learning Technologies, v. 3, n. 2, p. $91-95$.

Dietze, S. ., Sanchez-Alonso, S. ., Ebner, H. ., et al. (2013). Interlinking educational resources and the web of data: A survey of challenges and approaches. Program, v. 47, n. 1, p. $60-91$.

Fernandes, G. and Siqueira, S. (2013). Building users profiles dynamically through analysis of messages in collaborative systems. p. 75-82.

Fritzen, E., Siqueira, S. W. M. and De Andrade, L. C. V. (2012). Recuperação Contextual de Informação na Web para Apoiar Aprendizagem Colaborativa em Redes Sociais. XXIII Anais do Simpósio Brasileiro de Informática na Educação, v. 23, n. Simpósio Brasileiro de Informática na Educação, p. 26-30.

Heath, T., Singer, R., Shabir, N., Clarke, C. and Leavesley, J. (2012). Assembling and Applying an Education Graph based on Learning Resources in Universities. In Linked Learning (LILE) Workshop.

Pereira, C. K., Campos, F., Braga, R., Ströele, V. and David, J. M. N. (2014a). Elementos de Contexto em Sistemas de Recomendação no Domínio Educacional: um Mapeamento Sistemático. XIX Conferência Internacional sobre Informática na Educação, v. 19, p. 10.

Pereira, C. K., Campos, F., Ströele, V., David, J. M. N. and Braga, R. (2014b). Extracting User Profile and Context from Social Networks and Virtual Learning Environments to Recommend Educational Resources. Proceedings of the 13th International Conference WWW/Internet 2014, p. 171-178.

Pereira, C. K., Campos, F., Ströele, V., David, M. N. and Braga, R. (2014c). Extração de Características de Perfil e de Contexto em Redes Sociais para Recomendação de Recursos Educacionais. XXV SBIE 2014, v. 25, n. Cbie, p. 506-515.

Shani, G. and Gunawardana, A. (2011). Evaluating recommendation systems. Recommender systems handbook, p. 257-298.

Wohlin, C., Runeson, P., Höst, M., et al. (2012). Experimentation in Software Engineering. Springer Berlin Heidelberg.

Yin, R. K. (2001). Estudo De Caso - Planejamento E Metodos. p. 206 